\title{
On an effective gauge field description of a positron impurity in polarizable media
}

\author{
Document Version \\ Accepted author manuscript
}

Link to publication record in Manchester Research Explorer

\section{Citation for published version (APA):}

Arponen, J., Bishop, RF., \& Pajanne, E. (1987). On an effective gauge field description of a positron impurity in polarizable media. In P. Vashishta, R. K. Kalia, \& R. F. Bishop (Eds.), Condensed Matter Theories, Vol. 2 (pp. 373387). Plenum Publishing Corporation.

http://personalpages.manchester.ac.uk/staff/raymond.bishop/RFB_papers/[047] CMT_2(1987)373

\section{Published in:}

Condensed Matter Theories, Vol. 2

\section{Citing this paper}

Please note that where the full-text provided on Manchester Research Explorer is the Author Accepted Manuscript or Proof version this may differ from the final Published version. If citing, it is advised that you check and use the publisher's definitive version.

\section{General rights}

Copyright and moral rights for the publications made accessible in the Research Explorer are retained by the authors and/or other copyright owners and it is a condition of accessing publications that users recognise and abide by the legal requirements associated with these rights.

\section{Takedown policy}

If you believe that this document breaches copyright please refer to the University of Manchester's Takedown Procedures [http://man.ac.uk/04Y6Bo] or contact uml.scholarlycommunications@manchester.ac.uk providing relevant details, so we can investigate your claim.

\section{OPEN ACCESS}




\section{Condensed MATTER THEORIES volume 2}

Edited by

P. Vashishta and

Rajiv K.Kalia

Argonne National Laboratory

Argonne, Illinois

and

R. F. Bishop

University of Manchester Institute of Science and Technology Manchester, United Kingdom 
ISBN 0-306-42671-4

LC 87-656591

Proceedings of the 10th International Workshop on Condensed Matter Theories, held July 21-28, 1986, at Argonne National Laboratory, Argonne, Illinois

(C) 1987 Plenum Press, New York

A Division of Plenum Publishing Corporation 233 Spring Street, New York, N.Y. 10013

All rights reserved

No part of this book may be reproduced, stored in a retrieval system, or transmitted in any form or by any means, electronic, mechanical, photocopying, microfilming, recording, or otherwise, without written permission from the Publisher

Printed in the United States of America 


\title{
ON AN EFFECTIVE GAUGE FIELD DESCRIPTION OF A POSITRON IMPURITY
}

\section{IN POLARIZABLE MEDIA}

\author{
J. Arponen*, R.F. Bishop**, and E. Pajanne*** \\ *Department of Theoretical Physics, University of Helsinki \\ **Department of Mathematics, UMIST, Manchester \\ ***Research Institute of Theoretical Physics \\ University of Helsinki
}

\section{Abstract}

The local polarization around a positron impurity is described by a unitary operator, which defines a dynamical gauge field in interaction with the particle. We study the relation of this gauge field to the elementary collective excitations of the medium. We make contact with the generalized coherent bosonization scheme recently introduced in the extended coupled cluster theory, which suggests a definite parametrization of the polarization unitary operator in terms of a double similarity transformation. We derive the exact equations for the wavefunction and for the CCM amplitudes and show that they satisfy the conservation laws.

\section{Introduction}

The technique of positron annihilation at low energies has in the last 3 decades become much used in studying the properties of condensed materials, e.g. metals and their alloys ${ }^{1}$. In order to obtain a reliable interpretation for the experimental findings it is of great interest to find a first-principles theoretical description for the system composed of a positron, or generally a charged impurity, embedded in an electron medium. Although there exist rather advanced theoretical techniques e.g. for the case of a homogeneous electron gas, the extension of such formalisms to the general inhomogeneous case presents considerable difficulties. The two-component densityfunctional theory ${ }^{2}$ (DFT) can be used to formulate the concept of the positron wavefunction ${ }^{3-4}$, but the theory still has problems of two kinds: 1) There is no systematic way to go beyond the local density approximation (LDA); 2) Since the DFT wavefunction (i.e. the Slater determinant of the DFT orbitals) is not the true wavefunction, the amount of information, which can be extracted, is rather limited. 
The problem considered in the present article has a very general nature. It is a particular and relatively simple example of the field-theoretical one-body problem, and the present treatment is accordingly strongly fieldtheoretical in spirit. It was shown elsewhere ${ }^{5}$ and will be demonstrated more thoroughly in the present article that the theory of an impurity particle in a polarizable medium can be formulated as a special kind of gauge field theory, where one expresses the polarization of the electron medium in terms of an internal gauge field $A_{\mu}$. Our approach will be applicable not only to homogeneous electron systems, but also to the treatment of such inhomogeneous problems as e.g. surfaces and the localization problem, as well as to dynamical and transient problems.

The basic problem then consists of choosing a convenient parametrization describing the abstract internal gauge field operator and thus the displacement or polarization of the medium. In this article we shall show that a very convenient framework for the present problem is afforded by the well known coupled cluster method (CCM), which has been developed by Coester, Kümmel and coworkers in a series of fundamental papers ${ }^{5-8}$. We shall use the extended variational version of the $\operatorname{CCM}\left(\right.$ ECCM) ${ }^{9-10}$, which allows e.g. a consistent treatment of the dynamics and of the average values of physical observables in terms of the linked-cluster quasi-local subsystem correlation amplitudes.

\section{General definition of the impurity wave function}

We start with a general formulation of the impurity wavefunction. For the sake of simplicity we assume the interaction between the electron system and the positron to be spin-independent and thus omit the spin of the positron. As in our previous article (Arponen and Pajanne ${ }^{5}$ ), we def ine the state of the whole system, consisting of a positron embedded in the interacting electron medium, to be

$$
\left|\Psi_{1}\right\rangle=\int d^{3} r b_{r}^{\dagger} \chi(r t) U(r t)\left|\Psi_{0}\right\rangle,
$$

where $\mathrm{b}_{\mathrm{r}}{ }^{\dagger}$ is the positron creation field operator at the (three-dimensional) position vector $r,\left|\Psi_{0}\right\rangle$ is the true ground state of the electron system without the positron, $U(r t)$ is a unitary operator acting in the electron Hilbert space and $x(r t)$ can be interpreted to be the positron wave function, because $\left\langle\Psi_{1}\left|b_{r}^{\dagger} b_{r}\right| \Psi_{1}\right\rangle=|x(r t)|^{2}$.

In the coordinate-space representation the many-body wavefunction corresponding to the state (1) is of the form $\Psi\left(r ; x_{1} x_{2} \ldots x_{N}\right)=x(r) \psi_{r}\left(x_{1} x_{2} \ldots x_{N}\right)$, where $r$ is the positron coordinate and the $\left\{x_{i}\right\}_{i=1, \ldots, N}$ ar the electron coordinates, and the electron wavefunction $\psi_{r}\left(x_{1} x_{2} ., x_{N}^{\prime}\right)$ is required to be normalized for each $r$. Due to the strong screening correlations and electron density enhancement around the positron the electron wavefunction $\psi_{r}\left(x_{1} x_{2} \ldots x_{N}\right)$ depends on the coordinate $r$ in a crucial way, and therefore $x$ is to be understood as a quasi-wavefunction. In the independent particle model (IPM) the $r$-dependence of $\psi_{r}$ is ignored and the electron wavefunc- 
tion further represented as a slater determinant, for which reason e.g. the positron annihilation rate in metals becomes drastically underestimated. through

Next we define the internal gauge field $A_{\mu}(r t)=\left(A_{0}(r t),-\vec{A}(r t)\right)$

$A_{\mu}(r t)=i U^{\dagger}(r t)\left(\partial_{\mu} U(r t)\right)$

The covariant derivative $D_{\mu}=\left(D_{0},-\vec{D}\right)$ will then read

$D_{\mu}=\partial_{\mu}-i A_{\mu}=\partial_{\mu}+U^{\dagger}\left(\partial_{\mu} U\right)$

The time development of the state $\left|\Psi_{1}\right\rangle$ is given by the usual Schrödinger equation, which after premultiplication with $b_{r} U^{\dagger}(r t)$ can now be written as

$i \partial_{t} \chi(r t)\left|\Psi_{0}\right\rangle=\left[-\frac{1}{2 M} \vec{D}(r)^{2}-A_{0}(r t)-Z^{c}(r)+U^{\dagger}(r t) H^{e} U(r t)\right] \chi(r t)\left|\Psi_{0}\right\rangle$

Here $\mathrm{M}$ and $\mathrm{Z}$ are the mass and the charge number of the impurity, $\mathrm{V}^{\mathrm{C}}$ is the Coulomb potential operator (including the induced part) at the impurity position, and $\mathrm{H}^{e}$ is the Hamiltonian of the interacting pure electron system. Taking the scalar product of (4) with $\left\langle\Psi_{0}\right|$, we get the Schrödinger equation for $x(r t)$

i $\partial_{t} x(r t)=\frac{1}{2 M}\left[-\nabla_{r}^{2}-2 i \vec{\alpha}(r t) \cdot \nabla_{r}\right] x(r t)+v^{t o t}(r t) x(r t)$.

The total potential felt by the positron, $\mathrm{v}^{\text {tot }}$, is

$$
\begin{aligned}
V^{t o t}(r t)= & \frac{1}{2 M}\left\{\left\langle\Psi_{0}|\vec{A}(r t) \cdot \vec{A}(r t)| \Psi_{0}\right\rangle-i \nabla_{r} \cdot \vec{a}(r t)\right\}-\alpha_{0}(r t) \\
& -Z \int d^{3} x v(r-x)\left\langle\Psi_{0}\left|U^{\dagger}(r t) \rho^{e}(x) U(r t)\right| \Psi_{0}\right\rangle+z e \phi_{e x t}(r t) \\
& +\left\langle\Psi_{0}\left|U^{\dagger}(r t) H^{e} U(r t)\right| \Psi_{0}\right\rangle .
\end{aligned}
$$

In this equation $v(r-x)=e^{2} / 4 \pi \varepsilon_{0}|r-x|$ is the Coulomb potential, $\rho(r)$ is the total electron number density operator, and $\phi_{\text {ext }}(r t)$ is the external electrostatic potential. The third term in (6) is the interaction potential $\mathrm{ZV}^{\mathrm{C}}$ of the impurity with the electron system, and finally, $\alpha_{\mu}(r t)=$ $\left(\alpha_{0}(r t),-\vec{\alpha}(r t)\right)$ is the average of the gauge field $A_{\mu}$

$\alpha_{\mu}(r t)=\left\langle\Psi_{0}\left|A_{\mu}(r t)\right| \Psi_{0}\right\rangle=i\left\langle\Psi_{0}\left|U^{\dagger}(r t) \partial_{\mu} U(r t)\right| \Psi_{0}\right\rangle$.

The total potential in (6) looks formally simple. However, in reality it is very complicated; besides containing terms that arise from interaction with the unperturbed electron system, it also implicitly depends on the wavefunction $x$ itself, because the self-consistent polarization operator $U(r t)$ is coupled to $x$ by the requirement that the projections of equation (4) to other states than $\left|\Psi_{0}\right\rangle$ must also be satisfied. The equations (4)-(5) thus form a coupled set of equations in which $X$ depends on $U$ and $U$ depends on $x$ in an intricate fashion. 
From the definition in equation (2) we easily derive for the internal gauge field operator $A_{\mu}$ the following expression

$\partial_{\mu} A_{\nu}-\partial_{\nu} A_{\mu}-i\left[A_{\mu}, A_{\nu}\right]=0$,

or in component form

$\partial_{t} \vec{A}+\nabla A_{0}-i\left[A_{0}, \vec{A}\right]=0 \quad$ and

$\nabla \times \vec{A}+i \vec{A} \times \vec{A}=0$.

As a side remark we should mention that in the relativistic non-Abelian gauge field theories the gauge fields $A_{\mu}^{a}$ typically contain extra indices (a) related to the group structure of an internal symmetry group, and the commutator in equation (8) also involves the group commutator.

Furthermore, one can rewrite (2) to give a differential equation for $\mathrm{U}(\mathrm{rt})$ :

$\nabla U(r t)=i U(r t) \vec{A}(r t)$.

This can be integrated along some path in coordinate space to give U(rt) as a path-ordered phase factor

$U(r t)=P \exp \left\{-i \int_{r}^{\infty} d \vec{s} \cdot \vec{A}(s t)\right\}$,

Here we have assumed that the impurity is localized in some finite region of real space for which reason the operator $U(r t)$ at any given time $t$ can be assumed to satisfy the boundary condition $U(r t) \rightarrow I$ as $r \rightarrow \infty$.

Since the operator field $U(r t)$ is unique by definition, it follows that the integral in (12) cannot depend on the chosen particular path; therefore e.g. integration around any closed path gives the identity operator I. Nevertheless, path ordering in expression (12) is vital, because we cannot assume that the gauge field operators $\vec{A}(r t)$ commute with each other at different points.

On the basis of equation (12), the unitary operator U(rt), as well as the hermitean conjugate

$\mathrm{U}^{\dagger}(\mathrm{rt})=\mathrm{P} \exp \left\{-i \int_{\infty}^{\mathrm{r}} \mathrm{d} \overrightarrow{\mathrm{s}} \cdot \overrightarrow{\mathrm{A}}(\mathrm{s} t)\right\}$

can be considered as functionals of the abstract operator-valued gauge field $A_{u}$. The functional dependence is local in time, but not in space. Thus all the constituents of our theory, for example the potential $v^{\text {tot }}(r t)$ 
in the Schrödinger equation (5), are functionals of the gauge field $A_{\mu}$.

If the system experiences a nonzero classical external electromagnetic field $A_{\mu}(r t)^{e m}$, we have to modify the above notation and write for the total gauge field $A^{\text {tot }}$,

$$
A_{\mu} \rightarrow A_{\mu}^{\text {tot }}=A_{\mu}+\mathrm{eA}_{\mu}^{\mathrm{em}} \text {, }
$$

where te is the positron charge $(Z=1)$. Because the external field is now just a c-number field we can transform equations (9)-(10) with the familiar definitions for the magnetic induction $\vec{B}^{\mathrm{em}}$ and electric field $\overrightarrow{\mathrm{E}}^{\mathrm{em}}$ to read as

$$
\begin{aligned}
& \nabla \times \vec{A}^{\text {tot }}+i \vec{A}^{\text {tot }} \times \vec{A}^{\text {tot }}=\overrightarrow{\mathrm{B}}^{\mathrm{em}} \text { and } \\
& \partial_{t^{\mathrm{A}}} \overrightarrow{\mathrm{tot}}^{\text {tot }}+\mathrm{A}_{0}^{\text {tot }}-i\left[\mathrm{~A}_{0}^{\text {tot }}, \overrightarrow{\mathrm{A}}^{\text {tot }}\right]=-\overrightarrow{\mathrm{E}}^{\mathrm{em}} .
\end{aligned}
$$

If we consider external gauge transformations, which leave the external fields $\vec{E}$ and $\vec{B}$ invariant, we may assume the unitary operators $U(r t)$ and thus the internal gauge fields $A_{\mu}$ invariant. Then these gauge transformations will be associated with the phases of the wavefunction $x$ in standard fashion.

\section{Parametrization by expS theory using effective-action formalism}

As one notices from equation (1) the definition of the state $\left|\Psi_{1}\right\rangle$ contains the ground state of the interacting electron system $\left|\Psi_{0}\right\rangle$ modified by the local deformation $U$ caused by the impurity. The question now arises as to what would be the optimal way to parametrize the internal gauge field $A_{\mu}$ that describes the polarization of the electron medium. One way to do this systematically is to apply the ideas of the extended coupled cluster method (EECM) of references ${ }^{9-11}$ to this system. We start by defining a ketstate $\left|\Psi_{2}\right\rangle$ and a bra-state $\left\langle\Psi_{1}\right|$ for the system,

$\left|\Psi_{1}\right\rangle=\int d^{3} r b_{r}^{\dagger} x(r t) e^{S(r t)} e^{-S^{\prime \prime}(r t)}|\Phi\rangle$

$\left\langle\Psi_{1}^{\prime}\right|=\int d^{3} r\langle\Phi| e^{S^{\prime \prime}(r t)} e^{-S(r t)} \bar{x}(r t) b_{r} \cdot$

Here $|\Phi\rangle$ is an independent particle model (IPM) state. The amplitudes $S$ and $S "$ depend explicitly on the point $r$, where the impurity is located. This differs now from the previous definitions in equation (1) in the sense that the amplitudes $S$ and $S^{n}$ are now assumed to give both the correlation between the electrons as well as the local enhancement of charge around the positron. This means that in the limit $r \rightarrow \infty$, i.e. far out of the positron, both $S$ and $S^{\prime \prime}$ (or more precisly, the components $S_{i}$ and $S^{\prime \prime}{ }_{i}$, see below) should approach their values for the unperturbed electron system. Furthermore, we have now, because the ECCM approach makes use of a double similarity transform $\exp (S) \exp \left(-S^{\prime \prime}\right)$, a gauge field $A_{\mu}$ that is defined as 


$$
\begin{aligned}
A_{\mu} & =i e^{S "} e^{-S} \partial_{\mu}\left(e^{S} e^{-S "}\right) \\
& =i e^{S "}\left(\partial_{\mu} S\right) e^{-S "}-i\left(\partial_{\mu} S^{\prime \prime}\right),
\end{aligned}
$$

which is generally no longer hermitean.

We point out that the particular choice in equations (16)-(17) corresponds to definite gauge-fixing conditions for the internal gauge fields. This comes from the fact that the operators $S$ and $S "$ are genuine creation and destruction operators and do not contain any c-number terms. Therefore we have no freedom to impose further restrictions on the internal gauge fields such as e.g. the Coulomb-gauge condition $\nabla \cdot\langle\vec{A}\rangle=0$. One consequence of the present gauge-fixing parametrization is that the internal gauge field necessarily appears e.g. in the definition of the positron current (see next section) even if no external electromagnetic fields are present. Because the similarity transformations are not unitary, the wavefunctions $x$ and $\tilde{x}$ in this fixed internal gauge are not each other's complex conjugates.

The equations of motion for the positron wave function and for the amplitudes $S$ and $S^{\prime \prime}$ can be obtained in a concise ${ }^{9-11}$ manner by applying a variational principle to the action functional

$A=\int d t \int d^{3} r \int d^{3} r^{\prime}\langle\Phi| e^{S^{\prime \prime}(r t)} e^{-S(r t)} \tilde{x}(r t) b_{r}\left[i \partial_{t}-\left.H b_{r^{\prime}}^{\dagger} x\left(r^{\prime} t\right) e^{S\left(r^{\prime} t\right)} e^{-S^{\prime \prime}\left(r^{\prime} t\right.}\right|_{\Phi\rangle}\right.$

$\mathcal{A}=\mathcal{A}_{0}-\int \mathrm{dt} \overline{\mathrm{H}} ; \overline{\mathrm{H}}=\left\langle\Psi_{1} \cdot|\mathrm{H}| \Psi_{1}\right\rangle$

The most convenient parametrization from the point of view of diagram expansions involves a change from the original partial amplitudes $\left\{S_{i}, S^{\prime \prime}\right\}$ to new amplitudes $\left\{\sigma_{i}, \tilde{\sigma}_{i}\right\}$, and we write $9-11$

$S(r t)=\sum^{\prime} S_{i}(r t) c_{i}^{\dagger} \rightarrow \Sigma=\sum^{\prime} \sigma_{i}(r t) c_{i}^{\dagger}$

$S^{\prime \prime}(r t)=\sum^{\prime} S^{\prime \prime}{ }_{i}(r t) C_{i} \rightarrow \tilde{\Sigma}=\sum^{\prime} \tilde{\sigma}_{i}(r t) C_{i}$.

Here $\mathrm{C}_{i}$ and $\mathrm{C}_{i}{ }^{\dagger}$ are normalized even configuration operators, and the primed sum means that at least one particle-hole pair is annihilated or created $(i \neq 0)$. These new linked-cluster amplitudes $\sigma_{i}$ and $\tilde{\sigma}_{i}$ are now (quasi)local fields in the configuration space and they are given in terms of the amplitudes $S$ and $S^{\prime \prime}$ (Arponen et al. ${ }^{10-11}$ )

$$
\begin{aligned}
& \sigma_{i}(r t)=\left\langle\Phi\left|C_{i} e^{S "} S\right| \Phi\right\rangle, \\
& \tilde{\sigma}_{i}(r t)=S^{\prime \prime}{ }_{i}, \\
& S_{i}=\sum^{\prime} \sigma_{j} \bar{w}_{j-i},
\end{aligned}
$$

where $\bar{\omega}_{j-i}$ is the functional 
$\bar{w}_{j-i}=\left\langle\Phi\left|c_{i} e^{-S "} c_{j} \dagger\right| \Phi\right\rangle$

We postpone the precise description of the index set $\{i\}$ and the summation rules and, for the moment, keep the formalism completely general and thus applicable to an arbitary medium. For the general calculation rules we must refer to the original sources ${ }^{10-11}$. Using the above definitions one can now easily calculate the functional $\mathbb{t}_{0}$,

$\mathcal{A}_{0}=\iint \operatorname{dtd}^{3} r\left\{i \tilde{x} \dot{x}+i \tilde{x} \chi \dot{\sigma}_{0}-i \tilde{x} x\left[\dot{\sigma}_{j}^{\prime} \sigma_{j}\right\}\right.$, where

$\sigma_{0}(r t)=\left\langle\Phi\left|e^{S "} S\right| \Phi\right\rangle=-\sum^{\prime} \bar{\omega}_{i}(r t) \sigma_{i}(r t)$.

The functional derivatives of $\star_{0}$ with respect to the parameters are

$\frac{\delta t_{0}}{\delta \tilde{x}(r t)}=i \dot{x}(r t)+\left[i \dot{\sigma}_{0}-i \sum^{\prime} \dot{\tilde{\sigma}}_{j} \sigma_{j}\right] x$,

$\frac{\delta t_{0}}{\delta x(r t)}=-i \dot{\tilde{x}}+\left[i \dot{\sigma}_{0}-i \sum^{\prime} \dot{\tilde{\sigma}}_{j} a_{j}\right] \tilde{x}$,

$\frac{\delta A_{0}}{\delta \tilde{\sigma}_{i}(r t)}=i \tilde{x} x \dot{\sigma}_{i}+i(\dot{\tilde{x} x}+\tilde{\chi} \dot{x})\left(o_{i}-s_{i}\right)$,

$\frac{\delta A_{0}}{\delta \sigma_{i}(r t)}=-i \tilde{x} x \dot{\tilde{\sigma}}_{i}+i(\dot{\tilde{x}} x+\tilde{x} \dot{x}) \bar{\omega}_{i}$.

In the above equations and often later we have omitted the argument $r$, which is common to all the amplitudes $\sigma_{i}, \tilde{\sigma}_{i}$ and $\tilde{w}_{i}$. The expressions above are formally local with respect to the space point $r$, which appears just as an external common parameter.

\section{Average values of operators}

Let us now present the average values of some operators of the theory that will be needed later. We focus first on the positron density matrix, for which we may write

$$
\begin{aligned}
\left\langle b_{r}^{\dagger}{ }_{r^{\prime}}\right\rangle & =\left\langle\Psi_{1}{ }^{\prime}\left|b_{r}^{\dagger}{ }_{r^{\prime}}\right| \Psi_{1}\right\rangle \\
& =\tilde{x}(r t) x\left(r^{\prime} t\right) K\left(r, r^{\prime}\right), \text { where } \\
K\left(r, r^{\prime}\right) & =\left\langle\Phi\left|\hat{K}\left(r, r^{\prime}\right)\right| \Phi\right\rangle, \text { and } \\
\hat{K}\left(r, r^{\prime}\right) & =e^{S^{\prime \prime}(r t)} e^{-S(r t)} e^{S\left(r^{\prime} t\right)} e^{S^{\prime \prime}\left(r^{\prime} t\right)} \\
& =P \exp \left[-i \int_{r^{\prime}}^{r} d \vec{S} \cdot \vec{A}(s)\right]
\end{aligned}
$$

From the fact that $K(r, r)=1$ it follows at once that the impurity density 
is given by $\left\langle b_{r} t_{b_{r}}\right\rangle=\tilde{x}(r t) x(r t)$. The true positron current density is now obtained from the above equations

$$
\begin{aligned}
\vec{J}^{\mathrm{p}}(r) & =\left.\frac{i}{2 M}\left(\nabla_{r}-\nabla_{r^{\prime}}\right)\left\langle\mathrm{b}_{r}^{\dagger} \mathrm{b}_{r^{\prime}}\right\rangle\right|_{r=r^{\prime}} \\
& =\frac{i}{2 M}[(\nabla \tilde{\chi}) x-\tilde{\chi}(\nabla x)]+\frac{1}{M} \tilde{x} x \vec{\alpha},
\end{aligned}
$$

where $\vec{\alpha}=\left.i \nabla_{r} K\left(r, r^{\prime}\right)\right|_{r=r^{\prime}}=\langle\Phi|\vec{A}(r t)| \Phi\rangle$. The last term $\tilde{\chi} \times \vec{a} / M$ arises from the polarization of the electron medium and from our specific gaugefixing conditions.

The positron kinetic energy $\left\langle\mathrm{T}^{\mathrm{p}}\right\rangle$, which is an interesting quantity in positron physics, is found to be

$$
\begin{aligned}
\left\langle\mathrm{T}^{\mathrm{p}}\right\rangle & =\left.\frac{1}{2 \mathrm{M}} \int \mathrm{d}^{3} r \nabla_{r} \cdot \nabla_{r^{\prime}}\left\langle\mathrm{b}_{r}^{\dagger}{ }_{r^{\prime}}\right\rangle\right|_{r}=r^{\prime} \\
& =\frac{1}{2 M} \int d^{3} r\left\{\nabla \tilde{x} \cdot \nabla x+i \vec{\alpha} \cdot[(\nabla \tilde{x}) x-\tilde{x}(\nabla x)]+\vec{\alpha}^{2} \tilde{x} x+\Delta \tilde{x} x\right\}
\end{aligned}
$$

Here the average of the gauge field, $\vec{\alpha}(r t)$, and the fluctuation of the gauge field around its average, $\Delta$, are given as :

$$
\begin{aligned}
\vec{\alpha}(r t)= & \langle\Phi|\vec{A}(r t)| \Phi\rangle \\
= & i \sum^{\prime}\left\{\vec{\omega}_{j} \nabla_{j}+\left(\sigma_{j}-s_{j}\right) \nabla \tilde{\sigma}_{j}\right\} \text {. and } \\
\Delta(r t)= & \langle\Phi|\vec{A}(r t) \cdot \vec{A}(r t)| \Phi\rangle-\langle\Phi|\vec{A}(r t)| \Phi\rangle^{2} \\
= & \left.\nabla_{r} \cdot \nabla_{r^{\prime}} K\left(r, r^{\prime}\right)\right|_{r=r^{\prime}}-\vec{\alpha}(r t)^{2} \\
= & -\sum^{\prime} \sum^{\prime} L_{i j} \nabla_{i} \cdot \nabla \sigma_{j}+\sum^{\prime} \sum^{\prime}\left[\delta_{i j}+2 \sum^{\prime} L_{i k} \sigma_{k+j}\right] \nabla_{i} \cdot \nabla \tilde{\sigma}_{j} \\
& -\sum^{\prime} \sum^{\prime}\left[\sigma_{i+j}+\sum^{\prime} \sum^{\prime} \sigma_{i+k} L_{k l} \sigma_{l+j}\right] \nabla \tilde{\sigma}_{i} \cdot \nabla \tilde{\sigma}_{j} \cdot
\end{aligned}
$$

The quantity $S_{i}$ is defined in equation (24) and $L_{i j}$ is

$L_{i j}=L_{j i}=\sum^{\prime} \omega_{k+1} \bar{\omega}_{i-k} \bar{\omega}_{j-1}$.

As pointed out by Arponen et al. 10 these coefficients $\left(L_{i j}\right)$ have a wellunderstood diagrammatic interpretation in terms of the o's.

The additional expectation values that one needs to calculate the total potential of the positron $v^{\text {tot }}$ in $(40 a)$ are

$$
\begin{aligned}
& \left\langle v^{e p}\right\rangle=-2 \iint d^{3} x d^{3} y \tilde{x}(x)_{x}(x) v(x-y)\left\langle\rho^{e}(y)_{x}\right\rangle \\
& \left\langle H^{e}\right\rangle=\int d^{3} r \tilde{x}(r) x(r)\left\langle H_{r}^{e}\right\rangle
\end{aligned}
$$


$\alpha_{0}(r t)=\left\langle\left.\Phi\right|_{A_{0}}(r t) \mid \Phi\right\rangle=-i\left[\sum^{\prime}\left[\dot{\sigma}_{i} \bar{\omega}_{i}+\dot{\tilde{\sigma}}_{i}\left(\sigma_{i}-s_{i}\right)\right]\right.$,

where we use the notation

$$
\begin{aligned}
& \left\langle H_{r}^{e}\right\rangle=\left\langle\Phi\left|e^{S "(r)} e^{-S(r)} H^{e} e^{S(r)} e^{-S^{\prime \prime}(r)}\right| \Phi\right\rangle \\
& \left\langle\rho^{e}(y)_{x}\right\rangle=\left\langle\Phi\left|e^{S "(x)} e^{-S(x)} \rho^{e}(y) e^{S(x)} e^{-S^{\prime \prime}(x)}\right| . \Phi\right\rangle
\end{aligned}
$$

\section{Equations of motion for the wave functions}

The equations of motion for the wave functions $\tilde{x}$ and $x$ are straightforwardly obtained by writing down the stationary conditions for the action functional in equation (19) with respect to the variations $\delta \tilde{x}$ and $\delta x$. Without going into details we only give here the resulting schrödinger equations, which are

$$
\begin{aligned}
& i \dot{\chi}(r t)=-\frac{1}{2 M} \nabla^{2} \chi(r t)-\frac{i}{M} \vec{\alpha}(r t) \cdot \nabla \chi(r t)+v^{t o t}(r) \chi(r t), \quad \text { (40a) } \\
& -i \dot{\tilde{\chi}}(r t)=-\frac{1}{2 M} \nabla^{2} \tilde{\chi}(r t)+\frac{i}{M} \vec{\alpha}(r t) \cdot \nabla \tilde{\chi}(r t)+\tilde{v}^{t o t}(r) \tilde{\chi}(r t) . \quad \text { (40b) }
\end{aligned}
$$

The total potential $v^{\text {tot }}$ is

$v^{\text {tot }}(r)=-\frac{i}{2 M} \nabla \cdot \vec{\alpha}(r t)+\frac{\left(\vec{\alpha}^{2}+\Delta\right)}{2 M}-\alpha_{0}(r t)-z \int d^{3} y v(r-y)\left\langle p{ }^{e}(y){ }_{r}\right\rangle+\left\langle H_{r}^{e}\right\rangle$,

and $\tilde{V}^{\text {tot }}$ is identical with $\mathrm{V}^{\text {tot }}$, except for the change in sign of the first term on the right-hand side of equation (41).

The total potential in (41) seems to contain the time-derivatives $\dot{0}$ and $\tilde{\sigma}$ through $\alpha_{0}(r t)$ (cf. equation (38)), but these can be eliminated with the use of the respective equations of motion for $\sigma$ and $\vec{\sigma}$ presented in the following section (cf. equation (53)), and thus the Hamiltonians appearing in the Schrôdinger equations $(40 a, b)$ for $x$ and $\tilde{x}$ are truly independent of time derivatives. The problem at hand thus reverts to finding a reasonable approximation for the sets of parameters $\left\{\sigma_{i}, \tilde{\sigma}_{i}\right\}$.

If we now combine the two equations (40) in an obvious way, and note the definition in equation ( 31 ), we obtain the familiar continuity equation for the positron density $\rho^{p}=\tilde{\chi} x$,

$$
\frac{\partial \rho^{\mathrm{p}}(r t)}{\partial t}+\nabla \cdot \vec{J}^{\mathrm{p}}(r t)=0
$$


The calculation of the equations of motion for the CCM amplitudes proceeds analogously to the ideas presented in the previous section by finding the stationary conditions for the action functional in (19) with respect to variations $\delta \sigma_{i}$ and $\delta \tilde{\sigma}_{j}$. In this case, however, the needed functional derivatives of $\mathrm{H}$ with respect to $\sigma_{i}$ and $\tilde{\sigma}_{j}$ are rather cumbersome, although in principle straightforward to calculate. The variation $\delta \mathrm{H}$ can be written in the following form

$$
\begin{aligned}
\delta H= & \int d^{3} r \vec{J}^{p}(r t) \cdot \delta \vec{a}(r t)+\frac{1}{2 M} \int d^{3} r \rho^{p}(r t) \delta \Delta(r t) \\
& -z \iint d^{3} r d^{3} x \rho^{p}(r) v(r-x)\left\langle\delta \rho^{e}(x)_{r}\right\rangle+\int d^{3} r \rho^{p}(r)\left\langle\delta H_{r}^{e}\right\rangle
\end{aligned}
$$

Now we only have to express the variations $\delta \vec{\alpha}, \delta \Delta,\left\langle\delta \rho^{\mathrm{e}}\right\rangle$ and $\left\langle\delta \mathrm{H}^{\mathrm{e}}\right\rangle$ in terms of the variations $\delta \sigma_{i}$ and $\delta \tilde{\sigma}_{i}$ of the basic amplitudes and to use (43) in conjunction with the results in equations (28c) and (28d). Observing the continuity equation (42), these two equations $(28 c, d)$ are written in the form

$$
\begin{aligned}
& \frac{\delta A_{0}}{\delta \tilde{\sigma}_{i}}=i \rho^{p} \dot{\sigma}_{i}+i\left[s_{i}-\sigma_{i}\right] \nabla \cdot \vec{J}^{p} \\
& \frac{\delta A_{0}}{\delta \sigma_{i}}=-i \rho^{p} \dot{\sigma}_{i}-i \bar{\omega}_{i} \nabla \cdot \vec{J}^{p}
\end{aligned}
$$

In the actual calculations there will occur partial cancellations between $\delta A_{0}$ and the first term on the right-hand side of equation (43), because we find for the functional derivatives of this term :

$$
\begin{aligned}
& \frac{\delta}{\delta \tilde{\sigma}_{i}}\left\{\int \mathrm{d}^{3} r \vec{J}^{\mathrm{p}} \cdot \delta \vec{\alpha}\right\}=i\left(S_{i}-\sigma_{i}\right) \nabla \cdot \vec{J}^{\mathrm{p}}-i \vec{J}^{\mathrm{p}} \cdot \nabla \sigma_{i} \\
& \frac{\delta}{\delta \sigma_{i}}\left\{\int \mathrm{d}^{3} r \vec{J}^{\mathrm{p}} \cdot \delta \vec{\alpha}\right\}=-i \bar{\omega}_{i} \nabla \cdot \vec{J}^{p}+i \vec{J}^{\mathrm{p}} \cdot \nabla \tilde{\sigma}_{i}
\end{aligned}
$$

The equation of motion e.g. for the amplitude $a_{i}$ is now obtained by taking the functional derivative of 4 with respect to $\tilde{\sigma}_{i}$. Taking notice of (43), (44) and (46) we finally have :

$$
\begin{aligned}
& i \rho \rho^{p} \frac{d}{d t} \sigma_{i}=\frac{\delta}{\delta \tilde{\sigma}_{i}} \bar{K}, \text { where } \\
& \bar{K}=\left\langle v^{e p}\right\rangle+\left\langle H^{e}\right\rangle+\frac{1}{2 M} \int d^{3} r \rho^{p} \Delta,
\end{aligned}
$$

where the various terms are given in (34), (36) and (37). What appears in (48) is the convective time derivative

$$
\frac{\mathrm{d}}{\mathrm{dt}}=\frac{\partial}{\partial \mathrm{t}}+\frac{\vec{J}^{\mathrm{p}}}{\rho^{\mathrm{p}}} \cdot \nabla
$$


which describes the time rate of change for an observer moving with the positron. The positron velocity field is

$\vec{v}(r)=\frac{\vec{J}^{\mathrm{P}}(r)}{\rho^{\mathrm{p}}(r)}=\frac{i}{2 \mathrm{M}} \nabla \log \left(\frac{\tilde{\chi}}{\chi}\right)+\frac{1}{\mathrm{M}} \vec{\alpha}$

Analogous to equation (48) we have for $\tilde{\sigma}_{i}$ :

$-i \rho p \frac{d}{d t} \tilde{\sigma}_{i}=\frac{\delta}{\delta \sigma_{i}} \bar{K}$

We can use equations (48), (52) and (33) to eliminate the apparent timedependence of $\alpha_{0}(r t)$ in Eq. (38) and obtain

$\alpha_{0}(r t)=+\frac{1}{\rho} p \vec{J}^{p} \cdot \vec{\alpha}-\frac{1}{\rho} p \sum^{\prime}\left\{\bar{\omega}_{i} \frac{\delta \bar{K}}{\delta \tilde{\sigma}_{i}}+\left(S_{i}-\sigma_{i}\right) \frac{\delta \bar{K}}{\delta \sigma_{i}}\right\}$,

which now guarantees that the Hamiltonians in equations (40a-b) are truly independent of time derivatives. We stress that all the equations obtained above are exact. To be able to calculate the functional derivatives of $\bar{K}$, we must further specify the Hamiltonian of the medium, as will be discussed later on.

\section{Connection to the Lee-Low-Pines transformation}

In addition to the present impurity problem there exist many others, like e.g. the polaron problem and the meson-nucleus system, which all have typical common features. Therefore a method introduced for one problem often can be applied also in the other cases. A particularly convenient trick of transforming to the impurity rest coordinate system was introduced for the polaron problem by Lee et al. (LLP) ${ }^{12}$. Two of the present authors used this transformation earlier for the probiem of a positron in an homogeneous electron gas ${ }^{13}$. The LLP transformation is performed by the unitary operator

$U_{L L P}=\exp \left[i\left(\vec{P}-\vec{P}^{e}\right) \cdot \vec{r}^{p}\right]$

where $\overrightarrow{\mathrm{P}}^{\mathrm{e}}$ is the total-momentum operator of the medium (electron gas), $\vec{r}^{p}$ the position operator of the impurity, and $\vec{P}$ is a constant vector, which represents the total momentum of the translationally invariant eigenstate of the total system. The main effects of this transformation are: 1) The impurity coordinate $\vec{r}^{\mathrm{p}}$ becomes a "dead" variable and the impurity can be regarded to be fixed at e.g. $\left.\vec{r}^{p}=0,2\right)$ The Hamiltonian is reduced to describe only the medium in the presence of the fixed impurity center, but it obtains new recoil terms describing the impurity-induced interactions between the degrees of freedom of the medium.

The Lee-Low-Pines transformation to the impurity-centric description is a global one, and assumes global translational symmetry. Looking carefully at the equations of motion of the present article, it can be seen that the representations (1) or (16)-(17) for the total wavefunction 
actually perform a local (or differential) LLP transformation in which no global symmetry needs to be assumed. The transformed Hamiltonian is essentially the $K$ of equation (49), and the term containing the fluctuation $\Delta$ corresponds to the recoil terms. If the impurity density ${ }_{\rho}^{p}(r)=\tilde{\chi}(r) \chi(r)$ is constant, it factorizes away from the equations of motion (48), (52) for $\sigma$ and $\tilde{\sigma}$, and the impurity position parameter $r$ remains a constant external parameter. If, however, the density $\rho^{p}$ is not constant, like in the cases of a real lattice, nonstationary state, or spontaneous trapping, new terms containing $\nabla \log \left(\rho^{\mathrm{p}}\right)$ arise in the equations for $\sigma, \tilde{\sigma}$ from the variations of the recoil term. These new terms cannot be obtained - at least easily by the global LLP transformation.

It is interesting to note that from the point of view of the medium the positron enters only through the hydrodynamic variables $\rho^{p}$ and $\vec{J}^{p}$ describing classical average flow. The same is true in the opposite direction; the quantities affecting the impurity wavefunctions $\chi, \bar{x}$ can be interpreted hydrodynamically to be due to the physical fields and fluctuations describing the medium. The relative phases of the basic amplitudes $x, \tilde{x}$ or $\sigma, \tilde{o}$ are combined within each subsystem (i.e. the impurity vs. the medium) into such physical quantities, which have classical meaning.

As indicated above, the differential LLP transformation is adequate to describe spontaneous breaking of translational symmetry like in the case of spontaneous trapping. There have been suggestions that positron trapping might occur in low-density electron gas, but to our knowledge the present expectation is that it does not happen in the metallic density regime.

Generalized coherent bosonization and collective eigenmodes of the medium

Several authors have used the CCM to study the homogeneous electron gas $^{14-19}$. The extended version of the theory (ECCM), however, has not yet been applied to this problem, and therefore we shall remain rather sketchy about the general formalism, and devote more attention to a few limiting cases.

As is pointed out in references 10-11, the exponential similarity transformations of the ECCM theory can be regarded as a definite bosonization scheme, where the guidelines are sought from a careful analysis of the structure of perturbation diagrams. In contrast to the conventional bosonization schemes (like e.g. the Sawada bosons 13,17 ), which are based on isomorphisms of the Lie algebras, the ECCM bosonization is rather aimed at entirely eliminating the quantal interactions of the bosons to the ultimate extent that the bosons become classical in a definite sense ${ }^{10-11}$.

The collective eigenmodes are found by expanding the average value of the Hamiltonian in powers of $\delta \sigma_{i}, \delta \tilde{o}_{i}$ around the stable ground state, and by diagonalizing the leading (second-order) terms of the expansion. The 
result is formally a "Ginzburg-Landau" Hamiltonian

$H\left[\sigma_{i}^{0}+\delta \sigma_{i}, \tilde{\sigma}_{i}^{0}+\delta \tilde{\sigma}_{i}\right]=H\left[\sigma_{i}^{0}, \tilde{\sigma}_{i}^{0}\right]+\delta H$

$=\mathrm{E}^{0}+\sum \mathrm{E}_{\ell} \tilde{\mathrm{C}}_{\ell} \mathrm{C}_{\ell}+($ terms with $\mathrm{CCC}, \operatorname{CCCC}, \ldots)$.

The deviations $\delta \sigma_{i}, \tilde{\delta}_{i}$ are linear functions of the normal-mode amplitudes $C_{\ell}, \tilde{C}_{\ell}$ with coefficients that are given by the eigenvectors of the dynamical matrix ${ }^{10}$. The dynamics is still given by the equations

$$
i \tilde{C}_{\ell}=\delta \mathrm{H} / \delta \tilde{\mathrm{C}}_{\ell}, \mathrm{i} \dot{\tilde{\mathrm{C}}}_{\ell}=-\delta \mathrm{H} / \delta \mathrm{C}_{\ell},
$$

and the amplitudes $C, \tilde{c}$ can be considered to be the average values of ideal boson operators in the (bi-)coherent states of an ideal boson Hilbert space.

The average values of all other operators can as well be expanded in powers of $\mathrm{C}$ and $\tilde{\mathrm{C}}$. It is obvious that the leading (quadratic) terms of $\mathrm{H}$ determine the linear response of the system to arbitrary small perturbations. The higher-order terms (CCC, CCCC, ...) are related to large perturbations and non-linear phenomena. By the aid of the Poisson-bracket relations of Ref. 10 it is easy to show that the above formalism exactly satisfies all conservation laws not only in the linear but also in the non-linear regime.

Coming back to the positron impurity problem, we find that the deviations $\mathrm{C}$ and $\tilde{\mathrm{C}}$ become functions of the positron position $r$. For instance, the electronic energy becomes

$H^{e} \rightarrow H_{r}^{e}=E^{0}+\sum E_{\ell} \tilde{C}_{\ell}(r) C_{\ell}(r)+($ higher terms $)$.

Assuming that the medium would be in its ground state in the absence of the impurity, the amplitudes then behave, for a fixed index $\ell$, as $c_{\ell}(r), \tilde{c}_{\ell}(r) \rightarrow 0$ in the physical sense as $r \rightarrow \infty$. For an accurate description of the electron density enhancement the higher-order terms of the Hamiltonian, however, turn out to be important in the metallic density regime and at lower densities $1,5,13$.

The role of the interaction $\left(v^{e p}\right)$ is vital, because it is the driving term of the polarization of the medium; without it there would be no $r$-dependence in the amplitudes $\sigma_{i}(r)$ and $\tilde{o}_{i}(r)$, and the recoil $\Delta$ would be simply zero, and $\left\langle\mathrm{H}^{\mathrm{e}}\right\rangle=\mathrm{E}^{0^{i}}$. The full expression

$v^{e p}=-Z \iint d^{3} r d^{3} x \tilde{x}(r) x(r) v(r-x)\left\langle\Phi\left|e^{S "(r)} e^{-S(r)} \underset{x}{a}{ }_{x}^{\dagger} e^{S(r)}\right| \Phi\right\rangle$,

where $a_{x}^{\dagger}, a_{x}$ are the electron field operators, gives rise to the diagrams of Fig.1. Analogous diagram representations can be drawn for $\left\langle\mathrm{H}^{\mathrm{e}}\right\rangle$ and the recoil term. In constructing such expressions it will be useful to express the average value of a product of operators in terms of low-order functional derivatives of the average values of the factors ${ }^{10}$. 


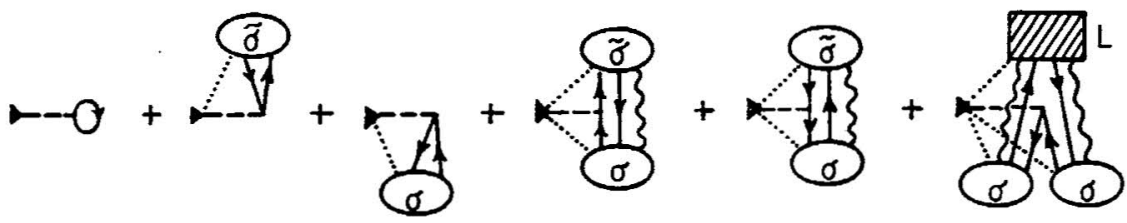

Eig.1 The ECCM diagrams for the electron-positron interaction. The small black triangle corresponds to the positron density factor $\tilde{\chi}(r) \chi(r)$; the dashed line is the Coulomb interaction, and the dotted lines are a mnemonic showing that the amplitudes $\sigma, \tilde{o}$ and $L$ depend on $r$. The wavy lines denote other possible electron-hole pairs.

$\underline{\text { Summary and discussion }}$

The general formalism presented above is an exact description for the positron motion in the polarizable medium. It satisfies the conservation laws, like e.g. the continuity equation, and the total energy conservation. For example, the slowing down of a fast positron in this picture takes place through emission of "classical" dissipative wave motion, or elastic waves, which disappear to infinity in a large system.

In the present article we have not considered specific approximations to the exact equations. The simplest truncation, SUB $1^{9}$, would correspond to the time-dependent Hartree-Fock approximation. This approximation becomes exact in the uniform limit, which is the limit of high electron density. The proper eigenexcitations are then the Sawada bosons, which in the lowmomentum regime can be treated classically and thus identified as the generalized coherent bosons. The other interesting limit is that of low electron density. The extreme case is the two-body problem where the ground state is the bound positronium (Ps) atom. Also this case is accurately treated by the SUB1 truncation. Now the non-linearity of the equations must be fully considered in contrast to the uniform limit where the linearresponse treatment is essentially sufficient.

As was emphasized earlier, all the observable properties of the system are in the ECCM method expressible through the quasi-local, linked-cluster subsystem correlation amplitudes $\{0, \tilde{0}\}$, which amplitudes may be interpreted as the average values of generalized coherent boson operators acting in a fictious ideal boson Hilbert space. The formalism preserves all the microscopic information in contrast to e.g. the density functional theory, where the obtained DFT orbital functions do not have close bearing to the true correlated many-body wavefunction.

The present representation of the many-body wavefunction was shown above to correspond to a local or differential form of the well-known Lee-Low-Pines transformation into the impurity-centric coordinate system. 
As a consequence of this, the equations for the medium contain recoil energy terms, which correspond to impurity-induced interactions between the degrees of freedom of the medium. The advantage of a local description is that it can be used in a conceptually simple way for translationally noninvariant systems like e.g. real metals and localization problems.

The formalism leads to separate, but coupled equations of motion for the impurity and for the medium. From the point of view of each of the subsystems, i.e. the impurity and the medium, the other subsystem enters only through classical or hydrodynamical variables. For the impurity system the theory provides a hydrodynamically complete and consistent description, in which all the conservation laws are fully satisfied.

\section{$\underline{\text { References }}$}

1. For review, see "Positron Annihilation", ed. by P.C.Jain, R.M.Singru \& K.P.Gopinathan, 1985, World Scientific Publ. Co., Singapore.

2. See e.g. "Theory of the Inhomogeneous Electron Gas", ed. by S.Lundqvist and N.H.March, 1983, Plenum Press, New York.

3. B.Chakraborty, Phys.Rev. B24: 7423 (1981).

4. B.Chakraborty and R.W.Siegel, Phys.Rev. B277: 4535 (1983).

5. J.Arponen and E.Pajanne, 1985, in Ref.1

6. F.Coester, Nucl. Phys. 7: 421 (1958).

7. F.Coester and H.Kümmel, Nucl. Phys. 17: 477 (1960).

8. For further references, see e.g. H.Kümmel, K.H.Lührmann and J.G.Zabolitzky, Phys. Rep. 36C: 1 (1978).

9. J.Arponen, Ann.Phys. 151: 311 (1983).

10. J.Arponen, R.F.Bishop, E.Pajanne and N.Robinson, to be published.

11. J.Arponen, R.F.Bishop and E.Pajanne, this volume.

12. T.D.Lee, F.E.Low and D.Pines, Phys. Rev. 90: 297 (1953).

13. J.Arponen and E.Pajanne, Ann. Phys. 121: 343 (1979).

14. D.L.Freeman, Phys. Rev. B15:5512 (1977).

15. R.F.Bishop and K.H.Lührmann, Phys. Rev. B17: 3757 (1978).

16. R.F.Bishop and K.H.Lührmann, Phys. Rev. B26: 5523 (1982).

17. J.Arponen and E.Pajanne, J.Phys. C15: 2665 (1982).

18. E.Pajanne and J.Arponen, J.Phys. C15: 2683 (1982).

19. K.Emrich and J.G.Zabolitzky, Phys. Rev. B30: 2049 (1984). 